\title{
Cancer in the thyroid is not always thyroid cancer
}

\author{
Katherine E. Akester ${ }^{1}$, Noel Somasundaram ${ }^{1}$, Salvador Diaz-Cano ${ }^{2}$, \\ Ashley B. Grossman ${ }^{1}$
}

Departments of ${ }^{1}$ Endocrinology and ${ }^{2}$ Histopathology, St. Bartholomew's Hospital, London EC1A 7BE, UK

\begin{abstract}
Most clinicians assume that a thyroid mass which appears to be malignant is most likely a tumour arising from thyroid cells. We present a case where the thyroid malignancy was associated with a degree of systemic clinical and biochemical disturbance which suggested alternative diagnoses, and in which fine-needle aspiration revealed that the patient had a thyroid lymphoma. Gastric biopsy showed that this was a primary gastric diffuse B cell lymphoma. Subsequent management provided a number of challenging and overlapping problems, and the patient eventually died from this high-grade malignancy. We review the prevalence, presentation, diagnosis and management of lymphomas presenting as thyroid masses, and underline the problems in management when there is multi-system disease.
\end{abstract}

Key words: thyroid, cancer, B cell gastric lymphoma

\section{INTRODUCTION}

There are approximately 12,000 new cases of thyroid cancer diagnosed per year in the USA. Over $90 \%$ are well-differentiated thyroid carcinomas, i.e. papillary or follicular, which carry a relatively good prognosis, although undifferentiated (anaplastic) thyroid cancer occurring in older age groups carries a poor long-term outlook ${ }^{1}$. However, when the clinician is presented with a firm thyroid mass, it is important to consider a wider differential diagnosis. Primary thyroid lymphoma is a rare entity usually occurring against a background of Hashimoto's thyroiditis ${ }^{1}$, while the thyroid is a not infrequent site of metastasis. We

Address correspondence and requests for reprints to: Professor A.B. Grossman, Department of Endocrinology, St. Bartholomew's Hospital, London EC1A 7BE, UK, Tel: +44207-6018343, Tfax: +44-207-6018505, E-mail:

A.B.Grossman@qmul.ac.uk

Received 23-08-03, Revised 20-09-03, Accepted 25-09-03 describe a patient who presented with a hard thyroid mass suggestive of a primary thyroid cancer, who proved to have metastatic lymphoma arising from a primary outside the thyroid.

\section{Case Report}

A 63-year old Somalian woman initially consulted her general practitioner in 2003 having noticed a swelling in her neck associated with voice change. She was clinically euthyroid and otherwise well, and was apparently reassured. However, 3 months later she was admitted to the Emergency Room systemically unwell: she complained of upper abdominal pain during the previous month accompanied by vomiting, anorexia, weight loss and constipation.

The patient had been investigated 6 months earlier for iron-deficiency anaemia, dyspepsia and constipation. A barium swallow and abdominal CT had revealed no abnormality, but the patient declined gas- 
troscopy and colonoscopy. Her current medication included treatment for hypertension and ischaemic heart disease following an inferior myocardial infarction in 1990. A thallium scan in 1999 had shown "unequivocal reversible ischaemia", but the patient declined the offer of coronary angiography. Clinical examination on admission revealed signs of anaemia; in addition, she had a hard thyroid mass and associated cervical lymphadenopathy. Her abdomen was distended but no organomegaly or ascites were present.

Investigation showed a microcytic anaemia $(\mathrm{Hb}$ $8.7 \mathrm{~g} / \mathrm{dl}, \mathrm{MCV} 78.7 \mathrm{fl}$ ) with normal haematinics but raised reticulocytes $(5.2 \%)$, and an elevated CRP (127 $\mathrm{mg} / \mathrm{L})$. Hyponatraemia $(133 \mathrm{mmol} / \mathrm{L})$ with elevated urea $(8.5 \mathrm{mmol} / \mathrm{L})$ and low bicarbonate $(18 \mathrm{mmol} / \mathrm{L})$ was also noted. Biochemical thyroid investigation revealed compensated hypothyroidism (free T4 17.0 pmol/L, normal range 11-28; TSH $9.6 \mathrm{mU} / \mathrm{L}$, normal range 1-4) with negative autoantibodies to thyroid peroxidase (TPO $11 \mathrm{IU} / \mathrm{ml}$ ), and an elevated serum thyroglobulin of $217 \mathrm{ng} / \mathrm{ml}$ (normal <50).

It was clinically suspected that she had a primary thyroid cancer, but the general systemic upset was discordant with this diagnosis. Ultrasound scanning (USS) revealed marked enlargement of the thyroid with a very heterogeneous echotexture and abnormal calcification accompanied by multiple abnormal lymph nodes with abnormal architecture and increased colour flow. The radiologist suggested that these appearances were highly indicative of a papillary carcinoma of the thyroid. However, two fine-needle aspirations for cytology of the thyroid revealed numerous polyclonal lymphocytes with some neutrophil polymorphs and plasma cells (Figure 1). No thyroid glandular tissue was observed. It was unclear whether the tissue sampled was unrepresentative and if a thyroid cancer had been missed, or was indeed a rare presentation of lymphoma. The patient refused core/open surgical biopsy and we therefore proceeded to CT scanning of her thyroid. This showed that the thyroid had almost entirely been replaced by a large inhomogeneous mass with mild enhancement slightly greater than muscle (Figure 2). A small focus of residual normal thyroid tissue remained in the upper left lobe; however, the abnormal thyroid mass extended superiorly, especially on the right to the level of the hyoid bone. Multiple enlarged lymph nodes were also detected on the right. These findings were consistent with thyroid lympho-

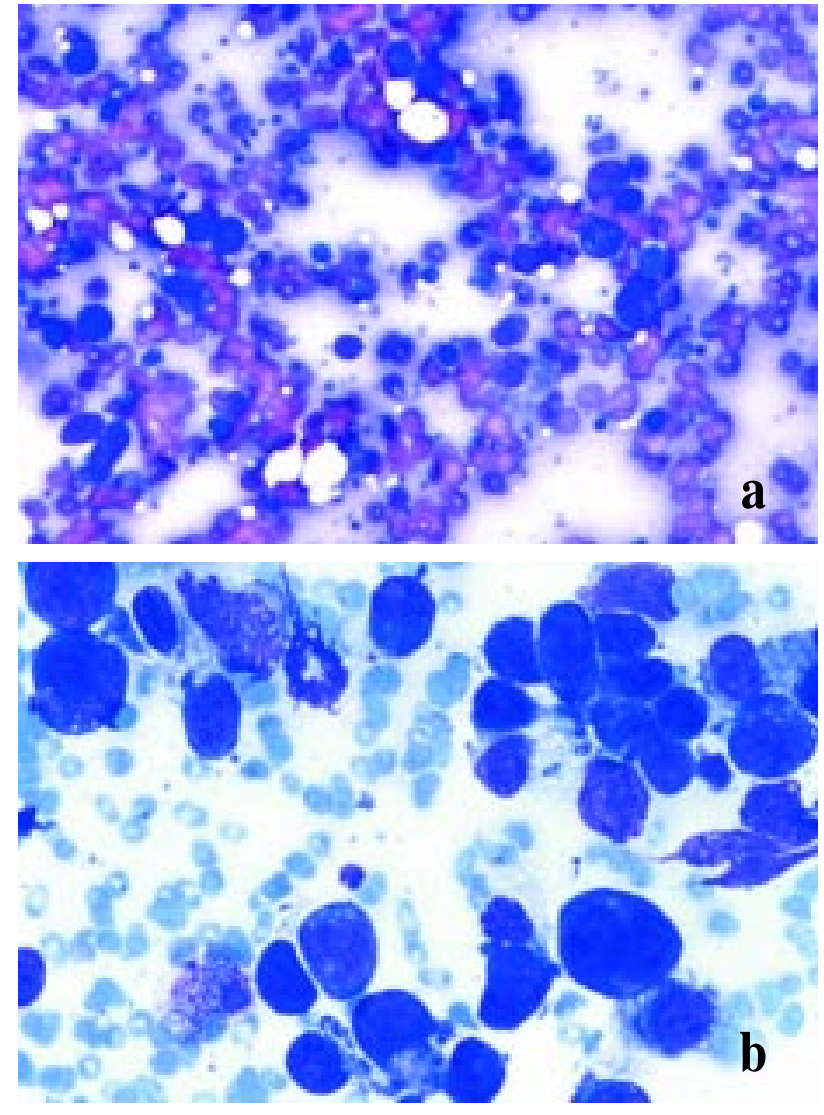

Figure 1. a. Fine needle aspirate of the thyroid showing variablysized lymphocytes in a haemorrhagic background. Occasional enlarged atypical lymphocytes are present (arrowhead) (Giemsa, $100 x)$. b. Small aggregates of these atypical lymphocytes are also seen in the smear (Giemsa, 400x).

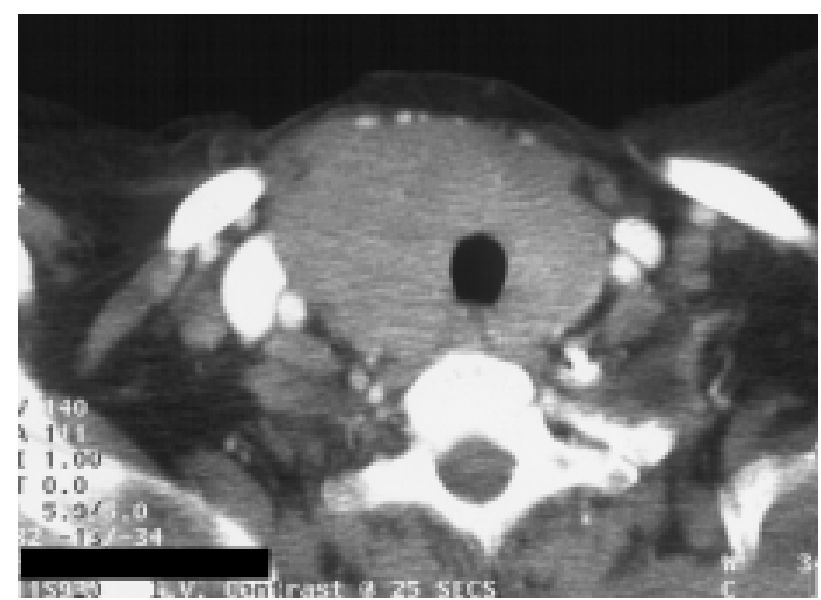

Figure 2. Contrast-enhanced CT of the thyroid. The thyroid gland has been almost entirely replaced by a large inhomogeneous mass, with enhancement characteristics slightly greater than muscle. A small focus of residual normal thyroid tissue is seen at the upper pole on the left. 
ma but other forms of primary thyroid cancer required exclusion. Again, biopsy was refused.

Chest CT indicated circumferential thickening of the thyroid and narrowing of the trachea, but no mediastinal lymphadenopathy was evident. In view of the abdominal symptoms, she was submitted to a abdominal CT of the abdomen which showed circumferential thickening of the stomach and blurring of the peripancreatic fat planes. There was also loss of the fat planes between the lesser curve of the stomach and the pancreas. However, no discrete masses or lymphadenopathy were observed. Gastroduodenoscopy revealed thickened gastric folds and multiple ulcers in the proximal and distal stomach. Gastric biopsies

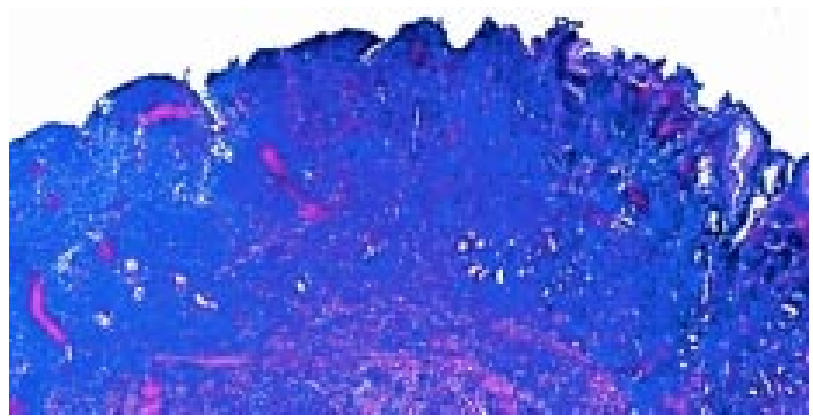

a

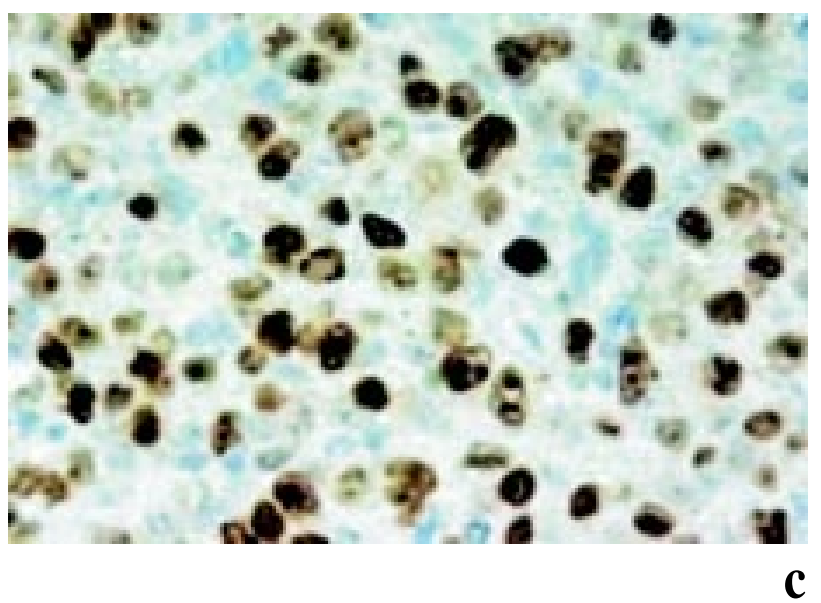

revealed that the tissue was almost completely replaced by large lymphoblastic cells which infiltrated between the glandular elements. No lymphoepithelial lesions were present, but neoplastic cells positive for CD10 and CD20, though negative for CD21 and BCL2, were present. The proliferation index as measured by Ki-67 was extremely high at 70-80\% (Figure 3 ). There was no evidence of extranodal lymphoma of mucosa-associated lymphoid tissue type (MALT). A diagnosis of diffuse large B cell lymphoma was made. Chest X-ray, echocardiogram, flexible sigmoidoscopy and $\mathrm{CT}$ of the pelvis were unremarkable.

The management of choice would have been to proceed to thyroid and lymph node biopsy to attain a

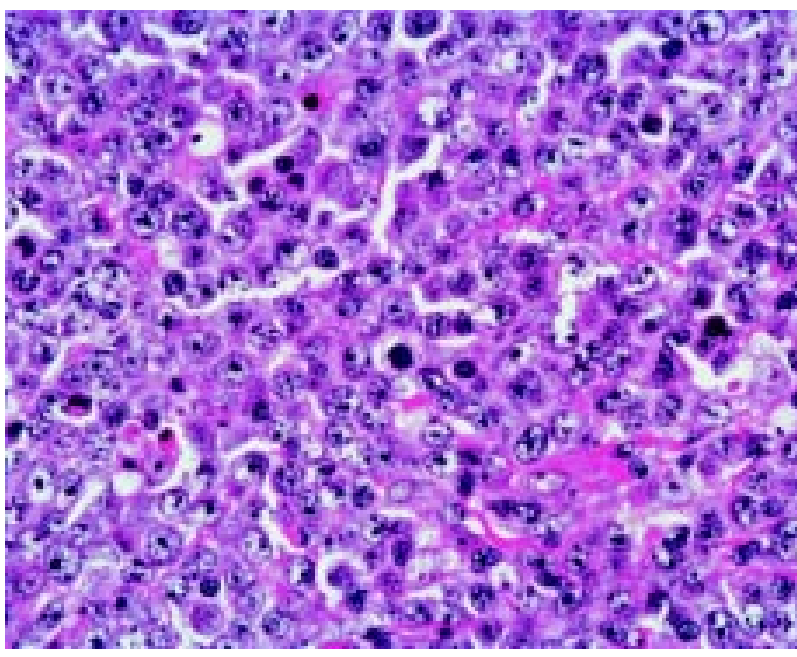

Figure 3. Gastric mucosa diffusely infiltrated by atypical lymphoid cells (a, H\&E 40x) with enlarged vesicular nuclei, prominent nucleoli, frequent mitotic figures (b, H\&E 400x), and a high Ki-67 index (70\%) (c, ABC Immunostaining 200x). The tumour cells express CD20 (B-cell marker) (d, ABC Immunostaining 400x). 
definitive thyroid diagnosis prior to treatment. However, yet again consent was refused. Regrettably, the patient discharged herself against medical advice after a fortnight with plans to undertake a pilgrimage.

The patient re-presented to the Emergency Room 11 days later, with a 2-week history of dysphagia to solids and chest pain at rest. Anorexia, weight loss, vomiting, malaise and constipation had continued. She had been refused as fit for travel by the airline and was now accompanied by her daughter. Her clinical signs were unchanged, but she now had more marked evidence of acidosis (bicarbonate $13 \mathrm{mmol} / \mathrm{L}$ ) and showed a deterioration in serum urea level $(9.6 \mathrm{mmol} /$ $\mathrm{L})$ and hypoalbuminaemia $(22 \mathrm{~g} / \mathrm{L})$. She had become biochemically hypothyroid (Free T4 9.7 pmol/L, TSH $28.8 \mathrm{mU} / \mathrm{L})$.

Fine-needle aspiration and cytology of the thyroid again showed lymphoid cells alone. No anaplastic or papillary cells were present. Thyroid USS was performed to reassess the size of the thyroid with regard to her dysphagia: this revealed a thyroid of $9 \mathrm{~cm}$ that now extended to just above the right subclavian artery. No dominant separate masses were observed. USS of the abdomen was normal with the exception of a small amount of fluid. Staging was completed with a negative bone marrow aspirate and trephine. Biochemical investigations showed that she continued to deteriorate and her lactate dehydrogenase was 1598 $\mathrm{U} / \mathrm{L}$, suggesting a rapidly progressive and extensive lymphoma with poor prognosis.

Naso-gastric feeding was commenced and following a baseline ECG liothyronine was slowly titrated to $2.5 \mu \mathrm{g}$ twice daily. It was planned to treat her with "CHOP" (cyclophosphamide, adriamycin, vincristine and prednisolone) chemotherapy and rituximab, but unfortunately, 11 days after admission the patient suffered a posterior/inferior myocardial infarction: liothyronine was temporarily stopped and full anticoagulation (enoxaparin $70 \mathrm{mg} \mathrm{bd}$ ) and antiplatelet therapy was commenced (clopidogrel $75 \mathrm{mg}$ od, aspirin 75 mg od). It was concluded that the patient could not be rendered euthyroid without cardiac intervention and therefore coronary artery stenting was proposed. Optimum cardiac status stability was also necessary prior to using a Hickman line as some of the chemotherapy agents initially suggested were cardiotoxic, further complicating the issue.
However, the patient's condition continued to deteriorate; she developed melaena and haemodynamic instability, and was transferred to the Intensive Care Unit. They were unable to site a central venous line due to the thyroid mass compressing the subclavian vein. She finally went into shock with both heart failure and acute renal failure. Despite intubation and ventilation she developed a further myocardial infarction from which she could not be resuscitated.

\section{DISCUSSION}

Our patient presented with a thyroid mass which eventually proved to be a metastasis from a lymphoma which also involved the stomach. Non-Hodgkin's lymphoma (NHL) classically arises in the lymph nodes: however, NHL may arise from extranodal sites where it can be classified as primary or secondary extranodal NHL. This patient had primary extranodal NHL as localised lymphoma appeared in extranodal tissues, whereas secondary NHL implies a predominantly node-based disease or widespread dissemination to extranodal tissues ${ }^{2}$. Extranodal manifestations of NHL have increased in the past two decades and now constitute between 24 and $48 \%$ of all $\mathrm{NHL}^{3}$. The gastrointestinal tract is the commonest site for extranodal disease ${ }^{2}$ with less than $3 \%$ affecting the thyroid gland ${ }^{1}$. Our patient therefore represents a relatively rare mode of presentation.

Multiple risk factors exist for developing extranodal lymphoma. Acquired immunodeficiency virus is the commonest cause of malignancy in unusual sites, with a 200 -fold excess in the expected NHL rate 3 . Our patient's only potential risk factor was being of Somalian origin, but HIV testing was declined by the patient. Immunosuppressive drugs, pesticides and solvents have also been implicated in addition to infections. This patient was negative for Hepatitis B, C and Epstein Barr virus.

Our patient presented initially with a rapidly developing thyroid swelling highly suggestive of a highgrade malignancy. Thyroid lymphoma classically affects elderly women with a 5:1 female to male preponderance ${ }^{1,4}$. This is concordant with the strong association of primary thyroid lymphoma and Hashimoto's thyroiditis ${ }^{1,5}$. The thyroid gland is naturally devoid of lymphoid tissue, and therefore it is thought either that Hashimoto's thyroiditis provides a lympho- 
cytic pool for future lymphoma development or that continual autoimmune stimulation predisposes to malignancy 60 - $60 \%$ of cases of thyroid lymphoma are associated with Hashimoto's thyroiditis, while $<1 \%$ of thyroiditis patients develop lymphoma ${ }^{1,7}$. Interestingly, Graves' disease is not a risk factor, weakening this autoimmune hypothesis ${ }^{1}$. Our patient developed lymphoma in the absence of a history of Hashimoto's thyroiditis, with negative antibodies to thyroid peroxidase (although the titre may fall with the development of lymphoma), while the abdominal pain, nausea, vomiting, weight loss and bleeding all suggested a non-thyroid primary lesion. These are, however, typical of a gastric lymphoma: $65 \%$ of gastrointestinal tract lymphomas arise in the stomach ${ }^{8}$.

Initial investigations showed compensated hypothyroidism. However, this gradually developed into hypothyroidism. This is a common finding and has no role in determining the diagnosis as both autoimmune destruction of the gland and replacement by lymphoma respectively produce a similar picture ${ }^{1}$. Thyroid peroxidase is helpful in diagnosing the former but is not definitive as both thyroiditis and lymphoid may coexist, particularly in MALT-variant tumours ${ }^{1}$. Fineneedle aspiration cytology (FNAC) is the initial investigation of choice as rapid results can be achieved. ${ }^{1,5}$ However, inconsistent results have been obtained for diagnosis of lymphoma. One study found an accuracy rate of $80 \%$ for diffuse large B cell lymphoma but only $40 \%$ for MALT due to the potential for thyroiditis to confuse $^{4}$. In others, FNAC was "suggestive but not diagnostic" in $50-60 \%$ of patients. ${ }^{5}$ In addition, the grade of malignancy cannot be established with FNAC. Our initial results showed lymphocytes; this suggested a differential diagnosis including thyroiditis, lymphoma, TB or a missed primary thyroid carcinoma.

Our patient declined core biopsy or open surgical biopsy which is usually required for definitive diagnosis and subtyping, 5 . The latter is superior as it provides larger, higher quality histological sections for histological analysis and lymphoma subtyping. Needle biopsies, although more convenient, do not necessarily represent the whole gland and can be confused with a thyroiditis lymphocytic infiltrate ${ }^{9}$. However, in this case the diagnosis of lymphoma was simpler as endoscopy and gastric biopsy diagnosed a diffuse large B cell lymphoma of the stomach. Therefore, the presence or absence of the thyroid lympho- ma in this case would not have altered the management. The pragmatic approach was to proceed to treatment without definitive knowledge of thyroid involvement. If thyroid lymphoma was present, as suspected, it would have shrunk relatively rapidly with chemotherapy, thus confirming the diagnosis.

As mentioned earlier, our patient had a diffuse large B cell gastric lymphoma which is the commonest form of primary gastric lymphoma and primary thyroid lymphoma ${ }^{2,4} .40 \%$ of gastric lymphomas are small B cell of MALT type, compared to $25 \%$ of thyroid lymphoma ${ }^{2}$. Although the thyroid is not part of the MALT system, lymphoid tissue can be acquired due to chronic immune system stimulation. There is an association of thyroid and gastric lymphoma of MALT type, possibly because they share the same endodermal origin; it is therefore interesting that large diffuse B cell lymphoma was the cause ${ }^{5,10,11}$. It may be that in some cases small cell MALT transforms to diffuse large B cell lymphoma histology ${ }^{2,5}$. We found no evidence of MALT, as indicated by the presence of no lymphoepithelial cells or CD10 positive cells. CD 20 positivity was also indicative of large diffuse B cell lymphoma ${ }^{2}$.

Small B cell MALT lymphomas are generally lowgrade and indolent, remaining localised. A primary thyroid lymphoma alone could be treated with radiotherapy and is associated with a high cure rate ${ }^{1}$. However, diffuse B cell lymphomas are highly aggressive and are associated with poorer prognosis ${ }^{1,2}$ requiring chemotherapy with/without additional radiotherapy $^{1,4,6}$. It is therefore important to use immunophenotypic and immunogenic analysis to determine the histological and immunologic classification as this dramatically affects management and prognosis ${ }^{1,2,4,5}$.

However, regrettably this woman's performance status on admission was only two (25-50\% of time spent in bed), and her international prognostic index for NHL was very poor. This was based on age 60, a serum LDH that was significantly high, a performance status of 2, stage IV disease and extranodal involvement $>1$ site $^{12}$. Our patient had the full set and therefore had an expected cure rate of only $44 \%$ with an overall 5-year survival rate of $26 \%{ }^{12}$. It would have been difficult to establish and maintain remission and this woman would probably have died within months, even with chemotherapy. 
This case, however, clearly indicates the difficulties associated with treating a patient with significant co-morbidity. Her ischaemic heart disease precluded rendering her euthyroid and the final lymphoma-related gastrointestinal bleed inhibited anticoagulation cover for angiogram and stent. Thus, her cardiac state was extremely vulnerable to the cardiotoxic chemotherapy regime which would have precipitated further problems had acute renal failure, heart failure and myocardial infarction not occurred.

\section{Conclusion}

This report highlights the importance of a broad differential diagnosis for suspected thyroid malignancy. Even high-grade diffuse B cell lymphomas are curable, if detected early. In addition, this case illustrates the importance of relating apparently unrelated signs from separate systems of the body. Finally, the challenge of therapeutic intervention in co-morbidity is evident. Not all cancers appearing in the thyroid arise from the thyroid cells and accurate tissue diagnosis is essential.

\section{REFERENCES}

1. Edmonds C 1997 Thyroid Lymphoma In: Sheaves R, Jenkins P, Wass J (eds) Clinical Endocrine Oncology, Black- well Science, London; pp, 131-133.

2. Salhany KE, Pietra GG, 1993 Extranodal lymphoid disorders. Am J Clin Pathol 99: 472-485.

3. Doll DC, 1999 Introduction: Extranodal Lymphomas. Semin Oncol 26: 249-250.

4. Sniezek JC, Holtel M, 2003 Rare tumours of the thyroid gland. Otolaryngol Clin North Am 36: 107-115.

5. Ansell SM, Grant CS, Habermann TM, 1999 Primary thyroid lymphoma. Semin Oncol 26: 316-323.

6. Austin JR, el-Naggar AK, Goepfert H, 1996 Thyroid cancers. II. Medullary, anaplastic, lymphoma, sarcoma, squamous cell. Otolaryngol Clin North Am 29: 611-627.

7. Larsen P, Davies TF, Hay ID 1998 The Thyroid Gland In: Wilson JD, Foster DW, Kronenberg HM, Larson P (eds) Williams Textbook of Endocrinology, W.B. Saunders Company, Philadelphia; pp, 495-496.

8. Crump M, Gospodarowicz M, Shepard FA, 1999 Lymphoma of the Gastrointestinal Tract. Semin Oncol 26: 324337.

9. Pinkus GS, 1987 Needle biopsy in malignant lymphoma. J Clin Oncol 14: 2415-2416.

10. Isaacson P, Wright DH, 1984 Extranodal malignant lymphoma arising from mucosa-associated lymphoid tissue. Cancer 53: 2515-2524.

11. Hyjek E, Isaacson PG, 1988 Primary B cell lymphoma of the thyroid and its relationship to Hashimoto's thyroiditis. Hum Pathol 19: 1315-1326.

12. Shipp MA, Harrington DP, Anderson JR, 1993 A predictive Model for Aggressive Non Hodgkin's Lymphoma. N Eng J Med 329: 987-994. 